\title{
Information Entropy Based on Propagation Feature of Node for Identifying the Influential Nodes
}

\author{
Linfeng Zhong $\mathbb{D}^{1}{ }^{1}$ Yu Bai, ${ }^{1}$ Yan Tian, ${ }^{2}$ Chen Luo, ${ }^{1}$ Jin Huang, ${ }^{1}$ and Weijun Pan $\mathbb{D}^{1}$ \\ ${ }^{1}$ Civil Aviation Flight University of China, Guanghan 618307, China \\ ${ }^{2}$ School of Science, Southwest Petroleum University, Chengdu 610500, China \\ Correspondence should be addressed to Weijun Pan; wjpan@cafuc.edu.cn
}

Received 24 February 2021; Accepted 9 May 2021; Published 20 May 2021

Academic Editor: Chenquan Gan

Copyright ( 12021 Linfeng Zhong et al. This is an open access article distributed under the Creative Commons Attribution License, which permits unrestricted use, distribution, and reproduction in any medium, provided the original work is properly cited.

For understanding and controlling spreading in complex networks, identifying the most influential nodes, which can be applied to disease control, viral marketing, air traffic control, and many other fields, is of great importance. By taking the effect of the spreading rate on information entropy into account, we proposed an improved information entropy (IIE) method. Compared to the benchmark methods in the six different empirical networks, the IIE method has been found with a better performance on Kendall's Tau and imprecision function under the Susceptible Infected Recovered (SIR) model. Especially in the Facebook network, Kendall's Tau can grow by $120 \%$ as compared with the original IE method. And, there is also an equally good performance in the comparative analysis of imprecise functions. The imprecise functions' value of the IIE method is smaller than the benchmark methods in six networks.

\section{Introduction}

The phenomena of spreading can be seen everywhere in nature [1-7]. Many activities can be described as spreading in nature [8-13]. In recent years, many research studies focused on the spreading process due to its theoretical meaning and practical value $[14,15]$, including rumor controlling [16-18], information diffusion [19-22], air traffic controlling [23-25], and viral marketing [26-28]. Among them, research on identification of the influential nodes in complex networks is a hotspot. Understanding the influence of the node has revealed new insight into applications such as mining the key nodes [29-34] and designing effective strategies to prevent epidemic from spreading or accelerate information diffusion.

The identification of influential nodes is of great significance in fields of epidemic and rumor control, targeted advertising, and air traffic planning $[35,36]$. Recently, many researchers have put forward a variety of centrality methods to deal with the problem in a more efficient way to identifying these nodes. Degree centrality can be regarded as a typical method to deal with the former problem in consideration for the local information $[37,38]$. In view of this idea, Chen et al. proposed the Local Rank method by considering the 4th order neighbors of the node [39]. By taking into account the location information of nodes in the network, through the $K$-shell decomposition method, Kitsak et al. [40] discovered a fact that the most influential nodes are located at the heart of the network. Then, a lot of improved methods based on the $K$-shell decomposition [41-43] have been proposed to identify the influential nodes. Closeness centrality [44] and betweenness centrality [45] are two path-based methods. In consideration of the neighbors' influence, Ren et al. [46] came up with the IRA method. Based on the IRA method, Zhong et al. [47] proposed the IIRA method by taking the propagation feature into account. Information entropy is also used as an important centrality to evaluate the influence of nodes $[48,49]$.

Most of the previous methods assume that the node's influence depends on its own importance. But there is another key factor that cannot be neglected, namely, the neighbors' importance. On the basis of this idea, Guo et al. [50] put forward a method of information entropy (IE) by considering the neighbors' information quantity. 
Nevertheless, the performance of the IE method is also affected by the propagation feature. In the example network that is presented in Figure 1, the influence of nodes 1 and 6 cannot be accurately identified by the IE method. In this case, we think that the neighbors' number and spreading rate are likely to have a positive effect on the target node. Based on this idea, we proposed an improved information entropy (IIE) method in which the target node's information entropy may be affected by the propagation feature. Compared with the benchmark methods in six real networks, the IIE method has been found with a better performance on Kendall's Tau and imprecision function under the Susceptible Infected Recovered (SIR) model $[51,52]$.

\section{IIE Method}

The original IE method assumes that the influence of the node should be obtained through the information entropy of its neighbors. In the IIE method, we argue that the spreading rate and the number of neighbors could adjust the initial information entropy. We can fulfill the identification of the influential nodes by using the final information entropy, namely, the IIE method. The details of the IIE method can be interpreted below.

In general, an undirected network $G=(N, E)$ can be described by an adjacent matrix $A=\left\{a_{i j}\right\} \in R^{n, n}$, where $N$ represents the number of nodes and $E$ represents the number of edges. If node $i$ is connected to node $j, a_{i j}=1$; if not, $a_{i j}=0$. And, we think that the spreading rate and the number of neighbors could adjust the target node's information entropy. Thus, the IIE value of any node $j$ can be calculated by

$$
\begin{aligned}
\mathrm{IIE}_{j} & =\sum_{i \in \Gamma(j)} \psi_{j} H_{i j}, \\
& =\sum_{i \in \Gamma(j)} \psi_{j}\left(-p_{i j} \log p_{i j}\right),
\end{aligned}
$$

where $H_{i j}$ is the information quantity provided from $i$ to $j$, $\psi_{j}$ represents the influence of the propagation feature, the spreading rate is $\beta$, and $k_{j}$ represents the number of neighbors for node $j$, also the expression of $k_{j}$ is

$$
k_{j}=\sum_{i \in G} a_{i j} .
$$

Equation (1) can be written as

$$
\mathrm{IIE}_{j}=\sum_{i \in \Gamma(j)}\left[1-(1-\beta)^{k_{j}}\right]\left(-p_{i j} \log p_{i j}\right),
$$

where $p_{i j}=\left(k_{i} / \sum_{m \in \Gamma_{L-i}} k_{m}\right)$ and $\Gamma_{L-j}$ indicates node $j$ 's $L$ th order neighbors. If $L=1$, it indicates node $j$ 's direct neighbors.

To describe the IIE method in more detail, we set $L=$ 1 and $\beta=0.2$ by taking into account the example network in Figure 2. For the black nodes (node 1), the improved information entropy (IIE) of node 1 is then calculated by $\operatorname{IIE}_{1}=\left(1-0.8^{4}\right) \times\left(H_{21}+H_{31}+H_{41}+H_{51}\right)=0.7734$.

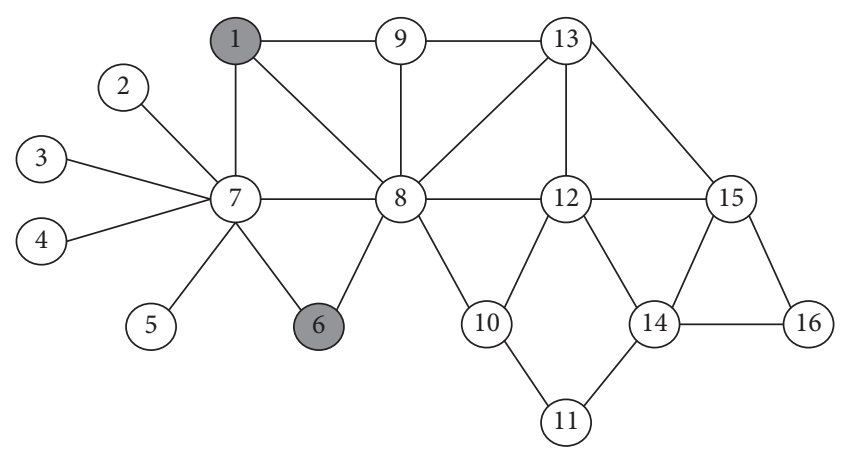

FIgUre 1: (Color online) The sample network in the figure has 16 nodes as well as 25 edges. The SIR model can be used to simulate the nodes' real spreading influence, that is, the amount of the infected nodes can be considered as spreading influence. We set $\beta=0.2$ and $\mu=1$, and the number of simulations are $10^{3}$. Therefore, we can get results of 2.405 and 2.101, respectively, for the spreading influence of nodes 1 and 6 . With the IE method, the influence of nodes 1 and 6 should be 1.875 and 2.023 , respectively.

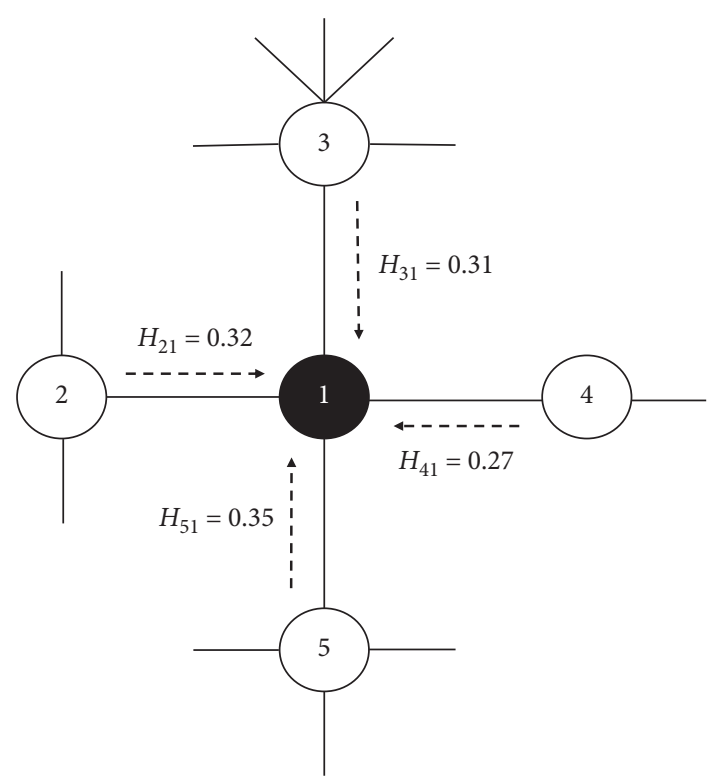

FIgure 2: (Color online) The figure shows how to calculate the IIE. There are four neighbors for node $1 . H$ is the information quantity. For example, $H_{21}$ represents the information quantity provided from node 2 to node 1 . We set $L=1$ and $\beta=0.2$. Each data point is calculated from the average of $10^{3}$ experiments.

\section{Results}

3.1. Data Description. There are six empirical networks used to evaluate the performance of the IIE method. The US air network [53] is an integral part of the US air traffic networks. The Polblogs network works as a network of political blogs in the United States with political relationship. The datasets are available on the web.

The e-mail network [54] refers to an electronic mail network of a university in Spain. The Soc-hamsterster network is a social network where the edges between nodes indicate the friendship or family ties. The Facebook network was derived from the Facebook online social platform, and 
its edges indicate the interpersonal relationship. The LastFM network [55] was derived from an FM broadcast platform for the Asian users where the edges represent that there exist friendships between nodes. The statistical attributes of the six networks are listed above in Table 1.

3.2. Measurement. For this paper, the node spreading influence is simulated with the SIR model [52]. There are three components to this system, namely, susceptible individuals $(S)$, infected individuals $(I)$, and recovered individuals $(R)$. In each time step of the SIR model, the susceptible neighbor nodes of each infected node will be infected randomly with a certain probability $\beta$. During this time, each infected node would recover with a certain probability $\mu$ and will no longer be infected. The spreading influence of a node is the range of infected nodes $X$ which refers to the number of nodes infected by the initial infected node in the whole network. The range of infected nodes $X$ was calculated from an average of $10^{3}$ experiments.

Kendall's Tau [56] and the imprecision function can be used to evaluate the superiority of the IIE methods. The value of Kendall's Tau is between $[-1,1]$, and this function can be used to evaluate whether there is a correlation between two ranking lists. The higher the value of Kendall's Tau, the stronger the correlation between the two ranking lists. Kendall's Tau $\tau$ can be expressed as

$$
\tau=\frac{2}{N(N-1)} \sum_{i<j} \operatorname{sgn}\left[\left(x_{i}-x_{j}\right)\left(y_{i}-y_{j}\right)\right] .
$$

$\operatorname{sgn}(x)$ works as a sign function; if $x>0$, the figure of $\operatorname{sgn}(x)$ equals to 1 ; if $x<0$, the figure of $\operatorname{sgn}(x)$ equals to -1 ; and, if $x=0$, the figure of $\operatorname{sgn}(x)$ equals to $0 . N$ represents the number of nodes in the lists, that is, in the network. Calculated by the centrality method, $x_{i}$ and $x_{j}$ are the order values in the ranking list for the nodes $i$ and $j$. And, $y_{i}$ and $y_{j}$ are the order values in the ranking list for the nodes $i$ and $j$ which are generated by the real spreading influence. If $\left(x_{i}-x_{j}\right)\left(y_{i}-y_{j}\right)>0$, it means that there is a large correlation coefficient between the two different ranking lists.

The imprecision function $\varepsilon_{\vartheta}(p)$ evaluates the performance of the centrality method by calculating the average propagation ability of the top key nodes in the ranking list obtained by the centrality method. $\varepsilon_{\vartheta}(p)$ should be expressed as

$$
\varepsilon_{\vartheta}(p) 1-\frac{M_{\vartheta}(p)}{M_{e f f}(p)}
$$

where $p$ is a proportion of the nodes to be selected, $p \in[0,1], N$ represents the number of nodes, $M_{\vartheta}(p)$ represents the average spreading influence of the top $p N$ nodes in the ranking list obtained by the centrality method, and $M_{e f f}(p)$ can be illustrated as the mean spreading influence of top $p N$ nodes in the ranking list calculated by the SIR model. If $M_{\vartheta}(p)$ is closer to $M_{e f f}(p), \varepsilon_{\vartheta}(p)$ is smaller. It means that the spreading influence for the top $p N$ nodes calculated by the centrality method is closer to the spreading influence of the top $p N$ nodes with the real ability of
TABLE 1: There are six fundamental statistical attributes in the six networks, such as $N, E,\langle k\rangle, \beta_{\text {thd }}, r$, and $C . N$ and $E$, respectively, represent the number of nodes and edges. $\langle k\rangle, \beta_{\text {thd }}, r$, and $C$, respectively, represent the average degree, epidemic threshold, degree assortativity, and clustering coefficient.

\begin{tabular}{lcccccc}
\hline Network & $N$ & $E$ & $\langle k\rangle$ & $\beta_{\text {thd }}$ & $r$ & $C$ \\
\hline US air & 332 & 2126 & 12.8 & 0.024 & -0.208 & 0.625 \\
Polblogs & 643 & 16097 & 7.09 & 0.052 & -0.217 & 0.232 \\
E-mail & 1133 & 5451 & 9.62 & 0.055 & 0.078 & 0.22 \\
Soc-hamsterster & 2426 & 16630 & 13.71 & 0.023 & 0.047 & 0.537 \\
Facebook & 4039 & 88234 & 43.69 & 0.009 & 0.063 & 0.605 \\
LastFM & 7624 & 27806 & 7 & 0.037 & 0.017 & 0.219 \\
\hline
\end{tabular}

Site $^{1}$.

spreading. This also indicates that the accuracy of the centrality method is higher.

3.3. Simulation Results. For this paper, we selected six real networks to test the IIE method. According to different networks, we set $\beta \in[0.1,0.2]$ and $\mu=1$ in the SIR model.

At first, we test the influence of different values of $L$ on the performance of the IIE method. $L$ represents the distance between nodes. If $L=1$, the direct neighbors' information quantity will be provided to the target node. And, if $L=2$, the target node's information quantity will be provided by its 2nd order neighbors. The influence of parameter $L$ on the IIE method in six networks is shown in Figure 3, $L \in[1,4]$.

From Figure 3, we can figure out that the effect of $L$ on Kendall's Tau $\tau$ calculated by the IIE method in different networks. Obviously, when we set the distance $L=2$, Kendall's Tau $\tau$ can get the maximum in the US air, Polblogs, e-mail, and LastFM networks. It demonstrates that the IIE method is more accurate than the ones generated by the other values of $L$ in the four networks. However, there are different phenomena in the Soc-hamsterster and Facebook networks. When $L=3$ or $L=4$, the value of Kendall's Tau is the largest, while the computation time of the IIE method increases dramatically. In addition, we know from the TDI theory [57] that individuals affect only a relatively small range of neighbors. Therefore, we set $L=2$ in later experiments.

To check the efficiency of the IIE method, the $K$-shell, degree centrality, closeness centrality, betweenness centrality, and IE method are selected as benchmark methods to compare with the IIE method in six networks. We set $\beta \in[0.1,0.2], \mu=1$, and the distance $L=2$. As can be seen from Figure 4, in the six networks, Kendall's Tau $\tau$ obtained by the IIE method is much bigger than the ones obtained by the benchmark methods. This indicates that the IIE method is superior to the benchmark method. It can also be seen from Figure 4 that, in the US air and LastFM networks, the value of Kendall's Tau $\tau$ obtained by the IIE method gradually increases along with the spreading rate $\beta$. On the contrary, in the Soc-hamsterster and Facebook networks, the value of Kendall's Tau $\tau$ obtained by the IIE method decreases with growth of spreading rate $\beta$. However, divergent phenomena exist in the Polblogs and e-mail networks. As the spreading rate $\beta$ increases, the value of Kendall's Tau $\tau$, 


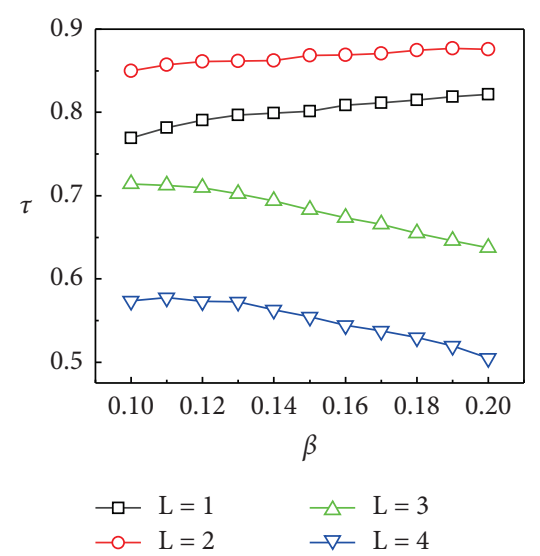

(a)

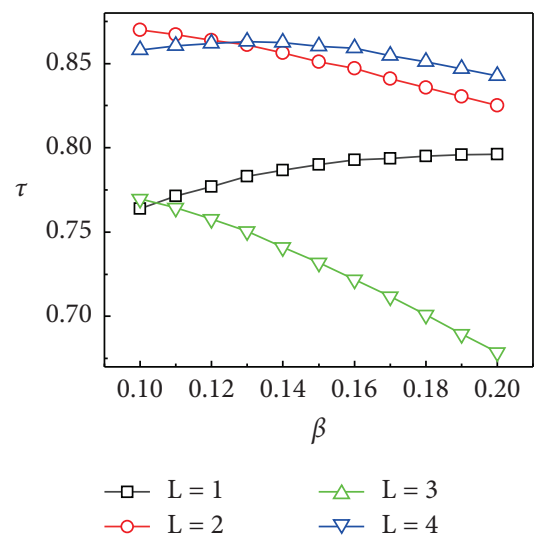

(d)

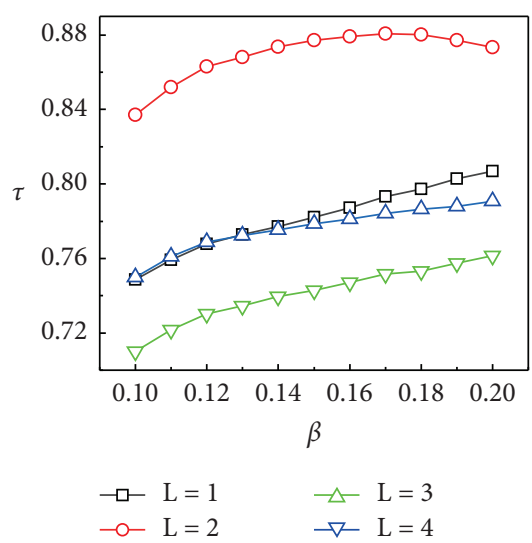

(b)

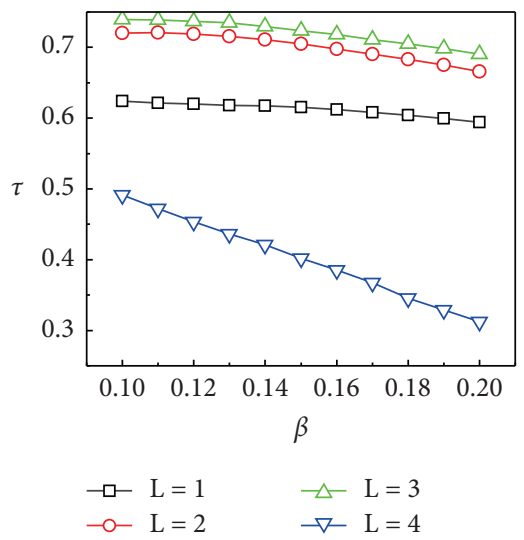

(e)

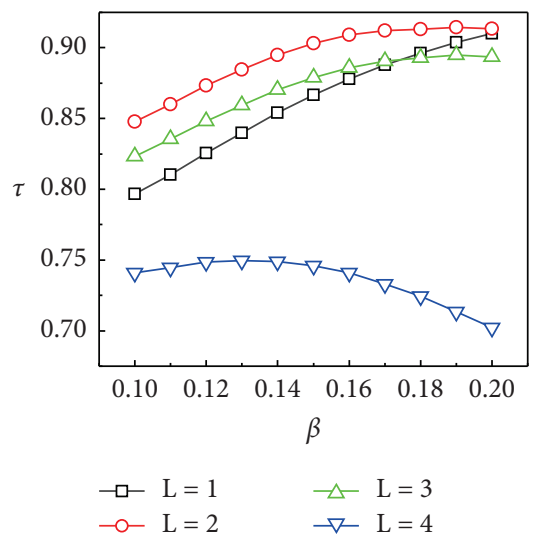

(c)

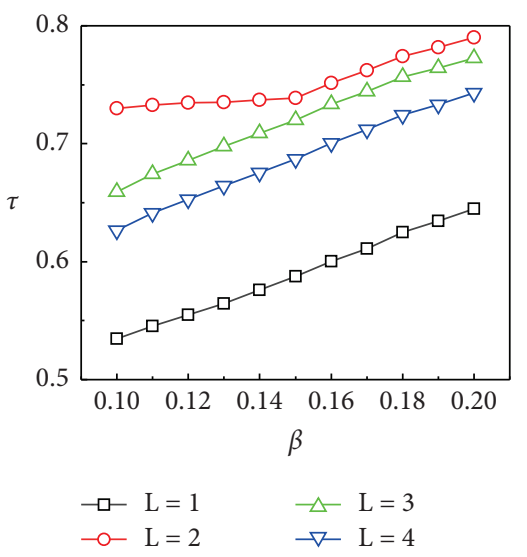

(f)

FIgUre 3: (Color online) The figure shows the effect of parameter $L$ on Kendall's Tau $\tau$ calculated by the IIE method in six networks. The larger value of Kendall's Tau indicates that the IIE method performs best along with the parameter $L$. We set $\beta \in[0.1,0.2]$ and $\mu=1$. Each data point is calculated from the average of $10^{3}$ experiments. (a) US air. (b) Polblogs. (c) E-mail. (d) Soc-hamsterster. (e) Facebook. (f) LastFM.

which is calculated obtained by the IIE method, will increase first and then decrease.

Figure 5 illustrates the improvement of ratio $\eta$ for Kendall's Tau as making a comparison between the IIE method and the benchmark methods. We define $\eta$ as

$$
\eta=\frac{\tau^{\mathrm{IIE}}-\tau^{0}}{\tau^{0}},
$$

where $\tau^{\mathrm{IIE}}$ represents Kendall's Tau which is obtained by the IIE method. $\tau^{0}$ represents Kendall's Tau $\tau$ calculated by the different benchmark methods. Obviously, if $\eta>0$, which means the performance of the IIE method is much better. Figure 5 clearly shows that, when the IIE method compared with the benchmark methods, Kendall's Tau $\tau$ increases considerably. That is, in the six networks, the IIE method is more accurate than the other benchmark methods on identifying the influential nodes. We can also find that, compared with the IE method, the maximum value of $\eta$ can grow by $80 \%$. Similarly, Kendall's Tau $\tau$ shows a significant increase when the IIE method is compared with the other benchmark methods in the US air network. This means that the IIE method is superior to the benchmark methods. The same phenomenon occurs in other different networks. In particular, in the Facebook network, compared with the IE method, the maximum value of $\eta$ can grow by $120 \%$ when $\beta=0.12$.

As can be seen from Figure 6, the imprecision functions $\varepsilon_{\vartheta}(p)$ of each method are presented and impressive results have been achieved by the IIE method in the six networks. In small networks such as US air and e-mail, the results of the IIE method are remarkably superior to those of other benchmark methods. For instance, $\varepsilon_{\text {IIE }}(p)$ is much lower than the benchmark methods, which means that the outcome of spreading predicted by the IIE method is more reliable than that predicated by the benchmark method. In the large LastFM network, $\varepsilon_{\text {IIE }}(p)$ is much lower than $\varepsilon_{\text {IE }}(p)$. This result reveals that the IIE method performs more accurately than the original IE method in identifying the most influential nodes. It is worth noticing that when $p$ is small, the IIE method shows much better performance than the other benchmark methods. These phenomena show the rationality of the IIE method considering the propagation feature of the target node. 


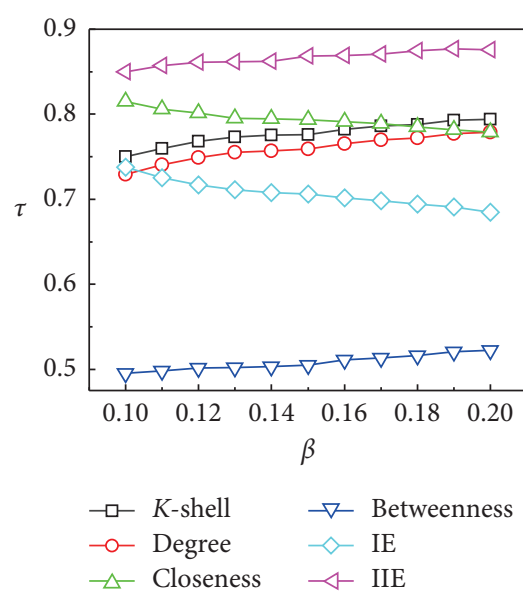

(a)

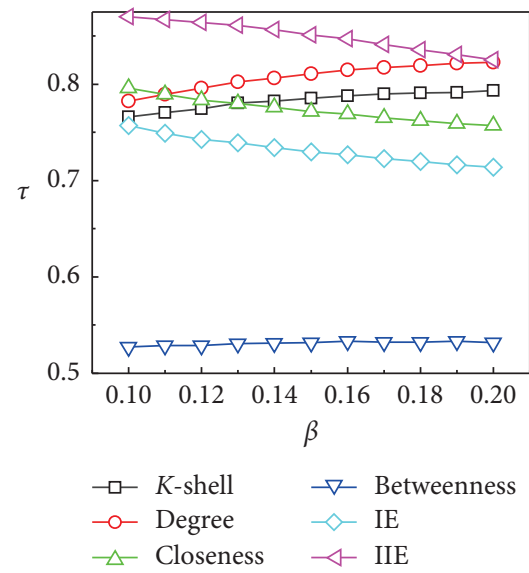

(d)

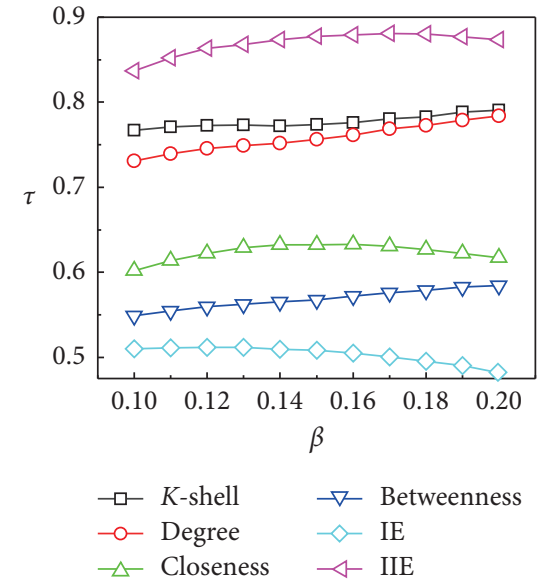

(b)

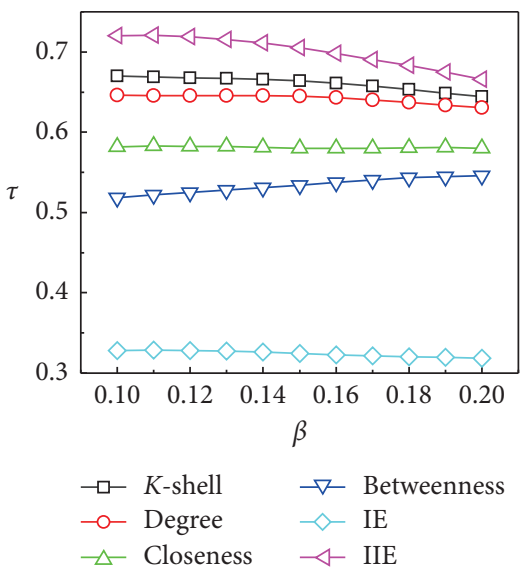

(e)

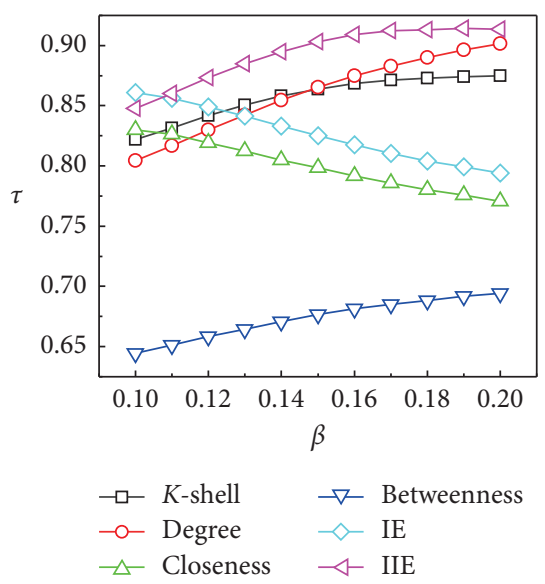

(c)

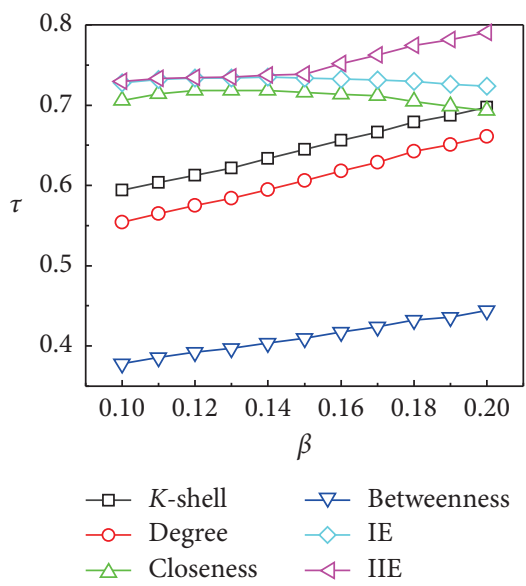

(f)

FIGURE 4: (Color online) The figure shows a comparison between the ranking list produced by the SIR model, and the ranking lists produced by the $K$-shell, degree centrality, closeness centrality, betweenness centrality, IE method, and IIE method lead to Kendall's Tau $\tau$. We set $\beta \in[0.1,0.2]$ and $\mu=1$. Each data point is calculated from the average of $10^{3}$ experiments. (a) US air. (b) Polblogs. (c) E-mail. (d) Sochamsterster. (e) Facebook. (f) LastFM.

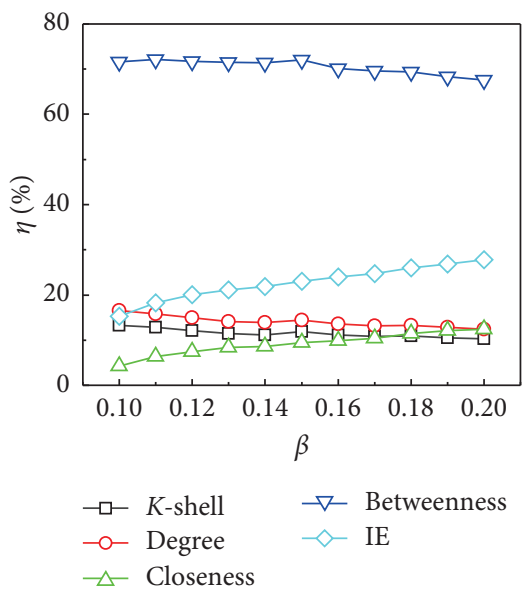

(a)

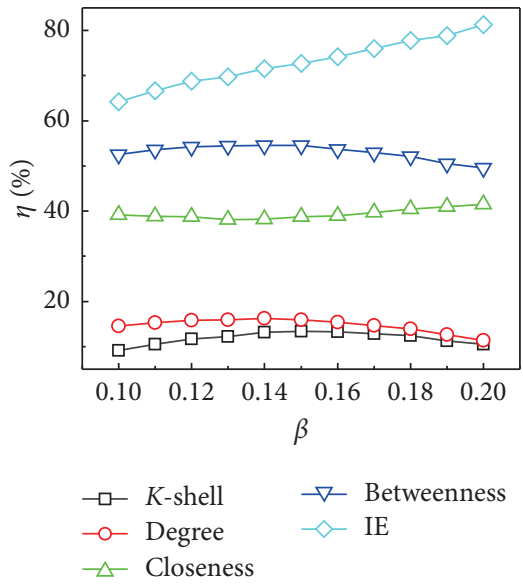

(b)

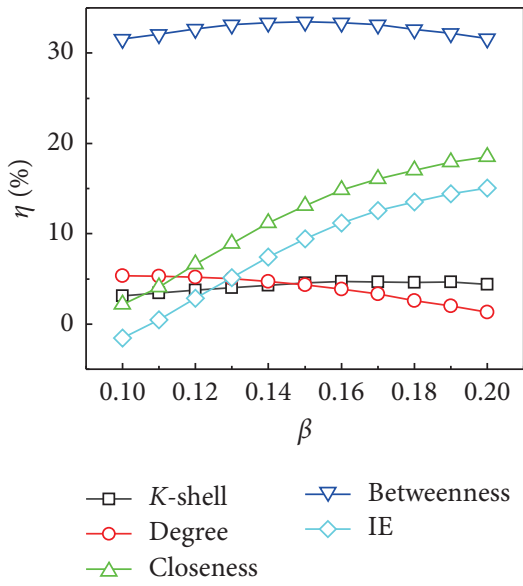

(c)

FIGURE 5: Continued. 


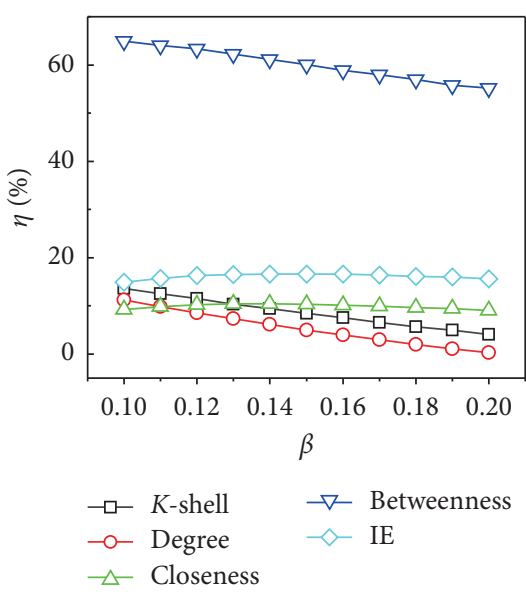

(d)

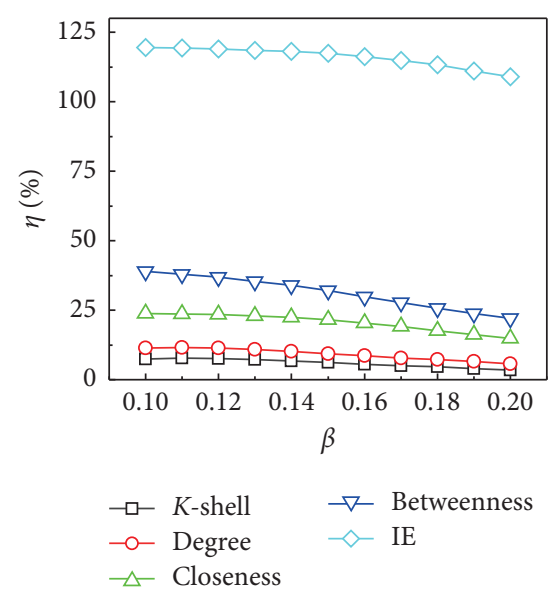

(e)

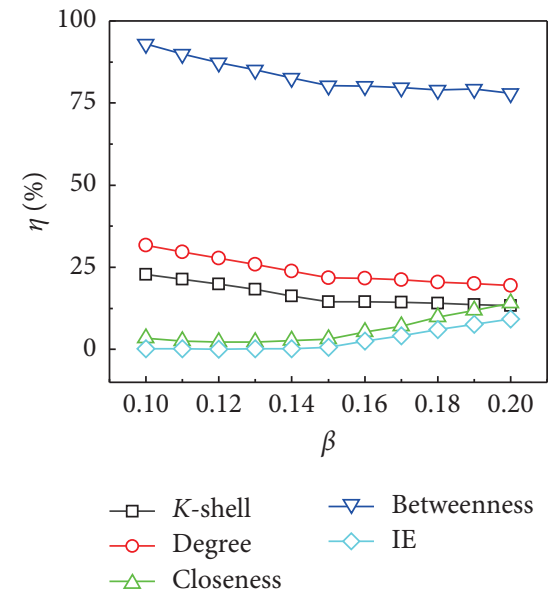

(f)

Figure 5: (Color online) The figure shows the improvement of ratio $\eta$ for Kendall's Tau as making a comparison between the IIE method and the benchmark methods in six networks. We set $\beta \in[0.1,0.2]$ and $\mu=1$. (a) US air. (b) Polblogs. (c) E-mail. (d) Soc-hamsterster. (e) Facebook. (f) LastFM.

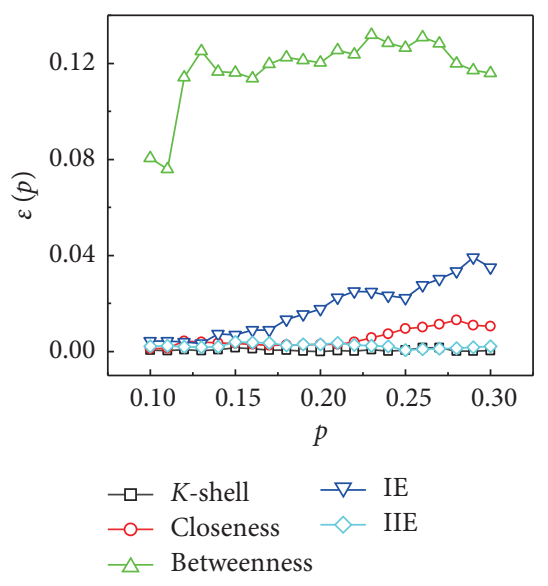

(a)

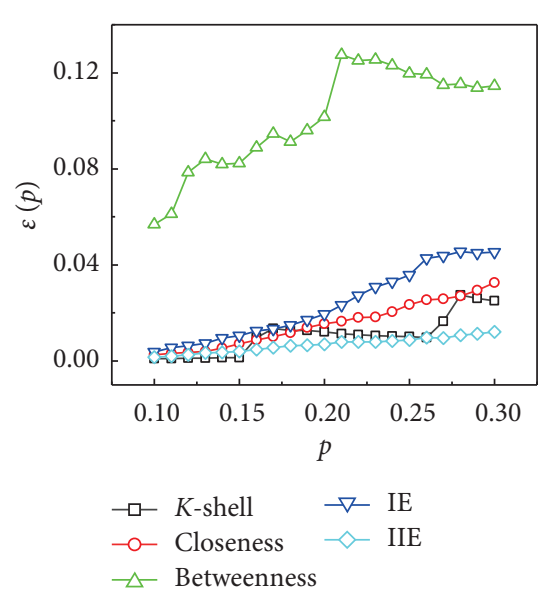

(d)

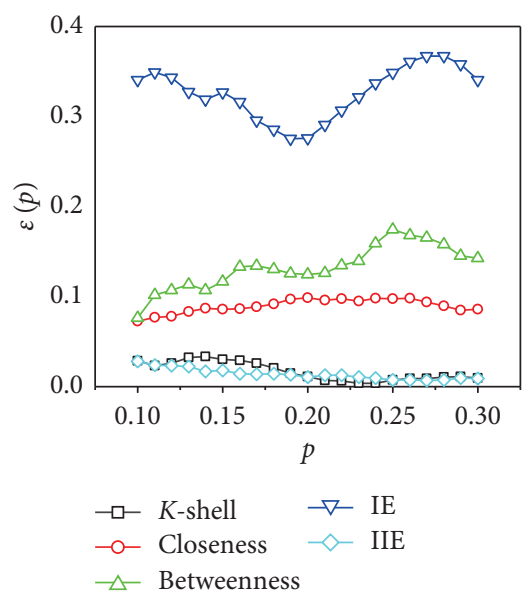

(b)

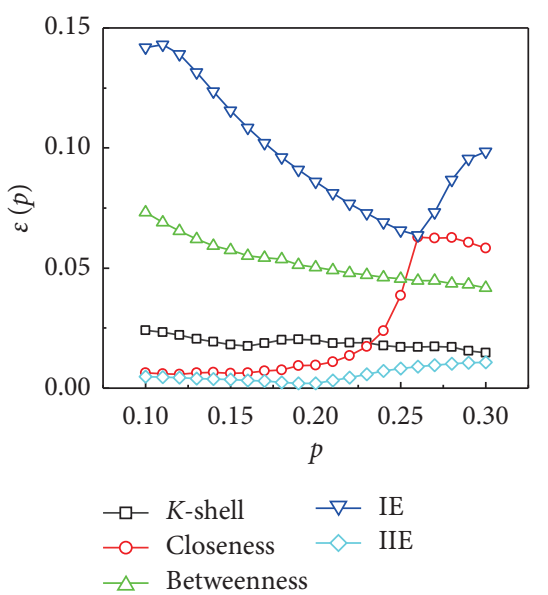

(e)

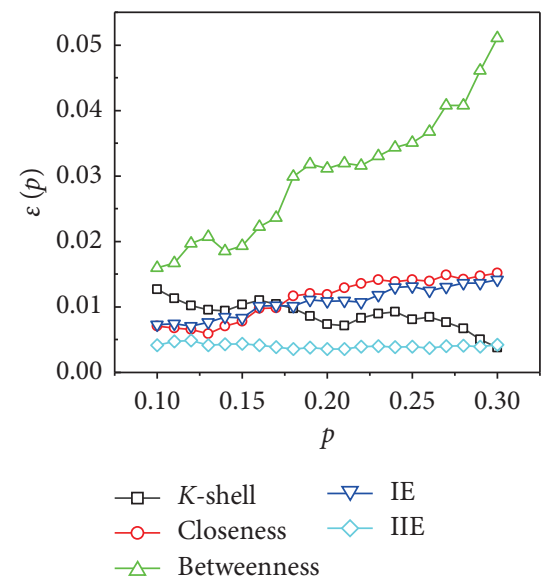

(c)

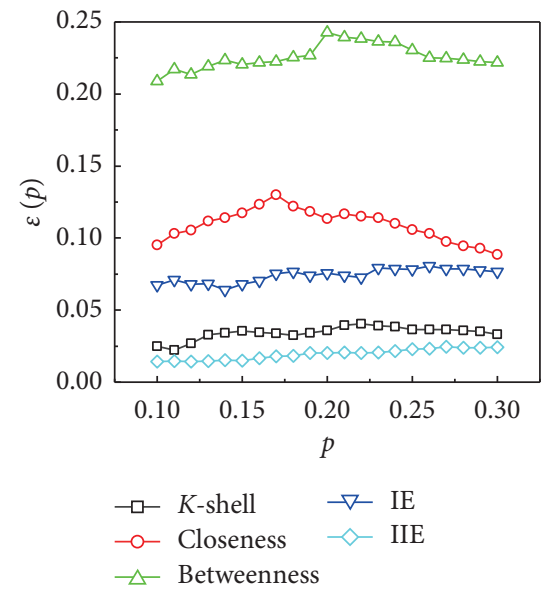

(f)

Figure 6: (Color online) This experiment compares different methods by using the imprecision functions $\varepsilon_{9}(p)$ in the six networks. $p$ is the proportion of selected nodes, $p \in[0,1]$. We set $\beta=0.2$ and $\mu=1$. The results were calculated from an average of $10^{3}$ experiments. (a) US air. (b) Polblogs. (c) E-mail. (d) Soc-hamsterster. (e) Facebook. (f) LastFM. 


\section{Conclusions}

For controlling the spreading process, one of the basic tasks is to estimate the spreading influence and identify the influential nodes. By considering the information entropy and spreading rate of the target nodes, we proposed an improved information entropy (IIE) method. The IIE method takes the spreading rate and the number of the target node's neighbors into account. And, those information dominate the new information entropy. According to the simulation results, the IIE method achieves a better performance than the IE method, and the IIE method $(O(N))$ does not add any parameters or increase computational complexity. In the six networks, the IIE method performs much better than the other benchmark methods, such as $K$-shell $(O(N))$, degree centrality $(O(N))$, closeness centrality $\left(O\left(N^{3}\right)\right)$, betweenness centrality $\left(O\left(N^{3}\right)\right)$, and IE method. Especially, in the Facebook network, comparing with the IE method, the maximum improved ratio $\eta$ goes up to $120 \%$. And, there also exists an equally good performance in the comparative analysis of imprecise functions. In the six networks, $\varepsilon_{\text {IIE }}(p)$ is much lower than the benchmark methods. These results demonstrate that the IIE method is sure to identify the influential nodes more precisely than the benchmark methods. And, the key component of the IIE method can be utilized by other centralities. For example, the information entropy of the IIE method can be also obtained by the neighbors' $K$-shell values.

Compared to the benchmark methods of the six networks, accuracy of the IIE method can be more satisfactory on identifying the influential nodes, while it poses some inevitable challenges. One of the challenges is that the IIE method merely takes the influence of the spreading rate for the target node into consideration and neglects the impact from target node's neighbors. The distance $L$ of the neighbors' should be paid more attention, for its value affects the performance of the IIE method. We should find out what factors affect the value of $L$. The temporal network has been paid more and more attention, which requires us to design an advanced information entropy method. And, it remains an interesting and open-ended problem.

\section{Data Availability}

The datasets used in the present study are available from the first author upon reasonable request (googlezlf@163.com).

\section{Conflicts of Interest}

The authors declare that they have no conflicts of interest.

\section{Acknowledgments}

This work was supported by the National Natural Science Foundation of China (no. U1733203), Safety Foundation of CAAC (no. AQ20200019), and Foundation of CAFUC (no. J2020-084).

\section{References}

[1] J. Ginsberg, M. H. Mohebbi, R. S. Patel, L. Brammer, M. S. Smolinski, and L. Brilliant, "Detecting influenza epidemics using search engine query data," Nature, vol. 457, no. 7232, pp. 1012-1014, 2009.

[2] C. Castellano, S. Fortunato, and V. Loreto, "Statistical physics of social dynamics," Reviews of Modern Physics, vol. 81, no. 2, pp. 591-646, 2009.

[3] D. Centola, "The spread of behavior in an online social network experiment," Science, vol. 329, no. 5996, pp. 1194-1197, 2010.

[4] R. Pastor-Satorras, C. Castellano, P. Van Mieghem, and A. Vespignani, "Epidemic processes in complex networks," Reviews of Modern Physics, vol. 87, no. 3, pp. 925-979, 2015.

[5] J. Wang and J. Wang, "Cross-correlation complexity and synchronization of the financial time series on potts dynamics," Physica A: Statistical Mechanics and Its Applications, vol. 541, Article ID 123286, 2020.

[6] J. Wang and J. Wang, "Measuring the correlation complexity between return series by multiscale complex analysis on potts dynamics," Nonlinear Dynamics, vol. 89, no. 4, pp. 2703-2721, 2017.

[7] J. Wang, J. Wang, and H. E. Stanley, "Multiscale multifractal dcca and complexity behaviors of return intervals for potts price model," Physica A: Statistical Mechanics and Its Applications, vol. 492, pp. 889-902, 2018.

[8] S. Boccaletti, G. Bianconi, R. Criado et al., "The structure and dynamics of multilayer networks," Physics Reports, vol. 544, no. 1, pp. 1-122, 2014.

[9] W. Wang, M. Tang, H.-F. Zhang, H. Gao, Y. Do, and Z.-H. Liu, "Epidemic spreading on complex networks with general degree and weight distributions," Physical Review E, vol. 90, no. 4, Article ID 042803, 2014.

[10] Z.-K. Zhang, C. Liu, X.-X. Zhan, X. Lu, C.-X. Zhang, and Y.-C. Zhang, "Dynamics of information diffusion and its applications on complex networks," Physics Reports, vol. 651, pp. 1-34, 2016.

[11] W. Wang, Q.-H. Liu, J. Liang, Y. Hu, and T. Zhou, "Coevolution spreading in complex networks," Physics Reports, vol. 820, pp. 1-51, 2019.

[12] Y. Lin, X. Wang, F. Hao et al., "Dynamic control of fraud information spreading in mobile social networks," IEEE Transactions on Systems, Man, and Cybernetics: Systems, vol. 2014, 14 pages, 2014.

[13] W. Wang, M. Tang, H.-F. Zhang, and Y.-C. Lai, "Dynamics of social contagions with memory of nonredundant information," Physical review e, vol. 92, no. 1, Article ID 012820, 2015.

[14] R. Albert and A.-L. Barabási, "Statistical mechanics of complex networks," Reviews of Modern Physics, vol. 74, no. 1, pp. 47-97, 2002.

[15] M. J. Keeling and P. Rohani, Modeling Infectious Diseases in Humans and Animals, Princeton university press, 2011.

[16] R. Pastor-Satorras and A. Vespignani, "Epidemic spreading in scale-free networks," Physical Review Letters, vol. 86, no. 14, Article ID 3200, 2001.

[17] R. Pastor-Satorras and A. Vespignani, "Epidemic dynamics and endemic states in complex networks," Physical Review E, vol. 63, no. 6, Article ID 066117, 2001.

[18] B. Gross and S. Havlin, "Epidemic spreading and control strategies in spatial modular network," Applied Network Science, vol. 5, no. 1, pp. 1-14, 2020.

[19] C. Liu and Z.-K. Zhang, "Information spreading on dynamic social networks," Communications in Nonlinear Science and Numerical Simulation, vol. 19, no. 4, pp. 896-904, 2014.

[20] K. M. A. Kabir, K. Kuga, and J. Tanimoto, "Analysis of SIR epidemic model with information spreading of awareness," Chaos, Solitons \& Fractals, vol. 119, pp. 118-125, 2019. 
[21] G. Miritello, E. Moro, and R. Lara, "Dynamical strength of social ties in information spreading," Physical Review E, vol. 83, no. 4, Article ID 045102, 2011.

[22] L. Pan, W. Wang, L. Tian, and Y.-C. Lai, "Optimal networks for dynamical spreading," Physical Review E, vol. 103, no. 1, Article ID 012302, 2021.

[23] D. Bertsimas and S. S. Patterson, "The traffic flow management rerouting problem in air traffic control: a dynamic network flow approach," Transportation Science, vol. 34, no. 3, pp. 239-255, 2000.

[24] K.-C. Pien, K. Han, W. Shang, A. Majumdar, and W. Ochieng, "Robustness analysis of the european air traffic network," Transportmetrica A: Transport Science, vol. 11, no. 9, pp. 772-792, 2015.

[25] K. Gopalakrishnan and H. Balakrishnan, "Control and optimization of air traffic networks," Annual Review of Control, Robotics, and Autonomous Systems, vol. 4, 2021.

[26] J. Leskovec, L. A. Adamic, and B. A. Huberman, "The dynamics of viral marketing," ACM Transactions on the Web, vol. 1, no. 1, p. 5, 2007.

[27] M. Kimura, K. Saito, R. Nakano, and H. Motoda, "Extracting influential nodes on a social network for information diffusion," Data Mining and Knowledge Discovery, vol. 20, no. 1, pp. 70-97, 2010.

[28] S. Gao, H. Wang, X. Yuan, and L. Lin, "Cooperative mechanism of sme growth in the mesoscopic structure with strategic and nonstrategic partners," IEEE Intelligent Systems, vol. 35, no. 3, pp. 7-18, 2019.

[29] S. Aral and D. Walker, "Identifying influential and susceptible members of social networks," Science, vol. 337, no. 6092, pp. 337-341, 2012.

[30] L.-F. Zhong, Q.-H. Liu, W. Wang, and S.-M. Cai, "Comprehensive influence of local and global characteristics on identifying the influential nodes," Physica A: Statistical Mechanics and Its Applications, vol. 511, pp. 78-84, 2018.

[31] S. Iyer, T. Killingback, B. Sundaram, and Z. Wang, "Attack robustness and centrality of complex networks," PloS One, vol. 8, no. 4, Article ID e59613, 2013.

[32] M. Bellingeri, D. Cassi, and S. Vincenzi, "Efficiency of attack strategies on complex model and real-world networks," Physica A: Statistical Mechanics and Its Applications, vol. 414, pp. 174-180, 2014.

[33] Z. Qiu, T. Fan, M. Li, and L. Lü, "Identifying vital nodes by achlioptas process," New Journal of Physics, vol. 23, no. 3, p. 033036, 2021.

[34] S. Wang, W. Lv, J. Zhang, S. Luan, C. Chen, and X. Gu, "Method of power network critical nodes identification and robustness enhancement based on a cooperative framework," Reliability Engineering \& System Safety, vol. 207, Article ID 107313, 2021.

[35] Y.-Y. Liu and A.-L. Barabási, "Control principles of complex systems," Reviews of Modern Physics, vol. 88, no. 3, Article ID 035006, 2016.

[36] J. You, J. Leskovec, K. He, and S. Xie, "Graph structure of neural networks," in Proceedings of the International Conference on Machine Learning, pp. 10881-10891, PMLR, Shenzhen, China, February 2020.

[37] M. G. Everett and S. P. Borgatti, "Extending centrality," Models and Methods in Social Network Analysis, vol. 35, no. 1, pp. 57-76, 2005.

[38] L. Lü, T. Lü, Q. M. Zhang, and H. E. Stanley, "The h-index of a network node and its relation to degree and coreness," Nature Communications, vol. 7, no. 1, pp. 1-7, 2016.
[39] D. Chen, L. Lü, L. Lü, Y.-C. Zhang, and T. Zhou, "Identifying influential nodes in complex networks," Physica a: Statistical Mechanics and Its Applications, vol. 391, no. 4, pp. 1777-1787, 2012.

[40] M. Kitsak, L. K. Gallos, S. Havlin et al., "Identification of influential spreaders in complex networks," Nature Physics, vol. 6, no. 11, pp. 888-893, 2010.

[41] A. Zeng and C.-J. Zhang, "Ranking spreaders by decomposing complex networks," Physics Letters A, vol. 377, no. 14, pp. 1031-1035, 2013.

[42] Y. Liu, M. Tang, T. Zhou, and Y. Do, "Core-like groups result in invalidation of identifying super-spreader by k-shell decomposition," Scientific Reports, vol. 5, no. 1, pp. 1-8, 2015.

[43] Y. Liu, M. Tang, T. Zhou, and Y. Do, "Improving the accuracy of the k-shell method by removing redundant links: from a perspective of spreading dynamics," Scientific Reports, vol. 5, no. 1, pp. 1-11, 2015.

[44] G. Sabidussi, “The centrality index of a graph," Psychometrika, vol. 31 , no. 4 , pp. 581-603, 1966.

[45] L. C. Freeman, "A set of measures of centrality based on betweenness," Sociometry, vol. 40, no. 1, pp. 35-41, 1977.

[46] Z.-M. Ren, A. Zeng, D.-B. Chen, H. Liao, and J.-G. Liu, "Iterative resource allocation for ranking spreaders in complex networks," EPL (Europhysics Letters), vol. 106, no. 4, Article ID 48005, 2014.

[47] L.-F. Zhong, J.-G. Liu, and M.-S. Shang, "Iterative resource allocation based on propagation feature of node for identifying the influential nodes," Physics Letters A, vol. 379, no. 38, pp. 2272-2276, 2015.

[48] T. Nie, Z. Guo, K. Zhao, and Z.-M. Lu, "Using mapping entropy to identify node centrality in complex networks," Physica A: Statistical Mechanics and Its Applications, vol. 453, pp. 290-297, 2016.

[49] X. Ai, "Node importance ranking of complex networks with entropy variation," Entropy, vol. 19, no. 7, p. 303, 2017.

[50] C. Guo, L. Yang, X. Chen, D. Chen, H. Gao, and J. Ma, "Influential nodes identification in complex networks via information entropy," Entropy, vol. 22, no. 2, p. 242, 2020.

[51] A. Barrat, M. Barthelemy, and A. Vespignani, Dynamical Processes on Complex Networks, Cambridge University Press, Cambridge, UK, 2008.

[52] M. E. Newman, "Spread of epidemic disease on networks," Physical Review E, vol. 66, no. 1, Article ID 016128, 2002.

[53] V. Batagelj and A. Mrvar, "Pajek-program for large network analysis," Connections, vol. 21, no. 2, pp. 47-57, 1998.

[54] J.-G. Liu, Z.-M. Ren, and Q. Guo, "Ranking the spreading influence in complex networks," Physica A: Statistical Mechanics and Its Applications, vol. 392, no. 18, pp. 4154-4159, 2013.

[55] B. Rozemberczki and R. Sarkar, "Characteristic functions on graphs: birds of a feather, from statistical descriptors to parametric models," in Proceedings of the 29th ACM International Conference on Information \& Knowledge Management, pp. 1325-1334, New York. NY. USA, October 2020.

[56] M. G. Kendall, "A new measure of rank correlation," Biometrika, vol. 30, no. 1/2, pp. 81-93, 1938.

[57] N. A. Christakis and J. H. Fowler, "The spread of obesity in a large social network over 32 years," New England Journal of Medicine, vol. 357, no. 4, pp. 370-379, 2007. 